vaccinate children much under six weeks, whereas the medical officer of the Privy Council has distinctly advised that children who are exposod to the influence of smallpox poison should be vaccinated within a week of their birth. It was also supposed to be dangerous to revaccinate elderly people, but this has been shown to be incorrect. There can be no question that grown-up people suffer, generally speaking, more from vaccination than children, the same as they do when attacked by measles, whoopingcough, \&c., and this I believe has given rise to the idea of vaccination affecting people worse during what is termed a varioloid state of atmosphere.

The materials used for collecting lymph are-1. Ivory points. 2. Glasses. 3. Capillary tubes. The last-named are the best, inasmuch as the lymph is always kept in a fresh state.

The methods employed for vaccinating are-1. Puncturing. 2. Scratching. 3. Blistering.

The last-mentioned (introduced by $\mathrm{Mr}$. Ellis, of London) is supposed to render the absorption of the lymph more certain, but it most undoubtedly entails a greater amount of trouble, and I cannot say from my own experience that it ensures a greater annount of success than either puncturing or scratching when carefully done. In a few instances, I have seen very bad arms result from this method. The eflects produced by revaccination are not, generally speaking, the same as those which exhibit thernselves after primary vaceination. $\Lambda \mathrm{s}$ far as I can see, mysclf, the eflect of the vaccine manifests itself in three different ways :-

1. 'There may be a perfect vesicle passing through all the different stages, showing that the protective eflects of the first vaccination have entirely passed away.

2 . There may be a scab formed, but no distinct vesicle, slowing that the protective effects of the first vaccination only partially remain.

3. 'There may be only slight redness produced, showing the protective effects of the first vaccination remain perfect, or nearly 80.

In all cases, I think, where there is not a perfect vesicle, revaccination should be tried agair, in order that the operation may not hereafter from carclessness fall into disreputc.

ln order that the general public may leain the bencfits resulting from vaccination and revaccination, all possible information should bo afforded them on the sub- ject, and medical men should be particularly anxious not to let any discredit fall upon the operation from a want of proper care. Once let the public be fully convinced of the fact that they cannot possibly receive harm from the inoculation of vaccine matter, and we may have the satisfaction of seeing smallpox in time banished from our shores.

\section{TWO CASES OF PARALYSIS OF THE THIRD NERVE.}

By C. K. Frske, M.D., St. Jolm, New Brunswick.

$\mathrm{C}_{\text {ASE }}$ I.-Mr. M- barrister, of this city, aged 40 , on the 16th of December, 1869, while driving, incautiously jumped from a sleigh, and, coming in collision with another rapidly-driven sleigh, received so severe a concussion as to render him insensible for several hours.

Ilis physician, Dr. Earle, was callod, and gave all needed attention to his case till restoration to consciousness, after which he complained of no pain or distress in the head, and there was no external contusion or wound upon the head or body, and he seemed all right with the exception of inability to raise the left eyelid.

On being called to him in consultation, the following day, I found a complete paralysis of the third nerve; closure of the left cyelid, on lifting which, I found the eye turned far to the left and slightly downward, by the combined action of the external rectus and superior oblique muscles; all motion of the eye suspended, the divergence being too great for double vision; the pupil dilated to about mid expansion; the accommodation power suspended, and withal giving the eyc a vacant stare; vision confused and uncertain as to distances, causing a staggering gait when attempting to walk. I found the patient in bed, quite comfortable; mind clear and memory good; pulse regular, and skin natural. Ile was required to remain in bed, and two or threo leeches were applied to the temple; and, believing there had been sanguincous effusion near the origin of the nerve, it was decided that rest and rather low diet should be persisted in for a fortnight or more. The Calabar bean was applied to the eye daily, and, after the end of a fortnight, faradization was applied in various ways two or three times a week. Some small blisters were applied over the brow and behind the car. 'The bean produced contraction of the pupil to some extent, but the iris did not readily come under its influence. 
The patient was allowed to walk about the house and to drive occisionally, and during the month of February warm baths were taken once or twice a week, and iodide of potassium was administered in medium doses three times a diay, the battery being applied two or three times a week; also the Calabar bean as at first till the 1 st of March, when there was a sudden and marked change in the case. On that day the functions of the nerve became nearly restored, the ptosis entirely relieved, and the inward motion of the eye became nearly perfect; vision improved rapidly, and the accommodation power was soon fully restored, the pupil being contracted to nearly its natural size without the aid of the bean.

'This case is remarkable for the completeness of the paralysis of the nerve and for the sudden restoration of its conducting power after so long a time-from the $16 \mathrm{th}$ of December till the 1st of March following.

Its record may be of interest to the practitioner, and assist in the prognosis of other cases of some wecks' standing, or even months.

This patient had been a "free liver," but readily consented to follow the advico of his pliysicians, and under a medium diet, with abstinence from stimulants, his general health and tone of body have much improved, and there is very little doubt the final cure was duc to these latter means, rather than to the use of the Calabar bean or the battery.

$\mathrm{C}_{A \mathrm{SE}}$ 11.-Mr. T55 , of spare habit, but rather full circulation, was suddenly attacked with ptosis of the left eyelid, attended with giddiness, on the 12 th of February, 1870.

He was brought immediately to my office, when I found the following appenrances: the left eyelid closed, which on lifting by my own hand, I found the pupil largely dilated, and the cycball turned much to the left and somewhint downward; no inflammation or pain, but he complained of vertigo.

On inquiring into the causes, I learned my patient had been, for many months, smoking large quantities of tobacco; a pipo in his moutl almost at all times, exeepting while eating or sleeping. I directed him to go home to his rooms and remain within-doors, to take efficient purgative medicines, and to abstain from smoking.

On the following day I found him much better, vertigo relieved, but with no improvement in the appearance of the eye and lid. I applied the Calabar bean, which readily contracted the pupil, but did not relieve the ptosis. I directed iodide of potassium to be taken in three-grain doses three times a day, and abstinence from the use of tobacco in all shapes.

In the course of a lortnight the eyesymptoms were much improved, and by the middle of March tho deformity was entirely removed, and at the present date the patient remains quite well.

The improvement in this case was gradual from day to day, while in the first case the change was sudden, after eleven weoks' duration, from complete ptosis, expansion of pupil, and strabismus divergens, to full restoration of the parts. $-N$. Y. Med. Jour.

\section{gablionraybicial âdoticrs.}

The Physiology and Pathology of Mind in the Lower Animals. By iV. LANofa Lindsay, M.I). lidinburgh. 1871.

Turs is an outline, in pumphlet, of a subject which the author has developed more fully in various British medical journals the past ycar. New and startling as the abovo theme may appear to those who have been accustomed to deny anything higher than puro instinct to the lower animals, much may be said for it. 'The old and arbitrary division of the sources of intelligent action into reason and instinct, reserving the former term to designate the higher cerebral operations of man, and confining all other animals to the use of the latter, must give way before the advance of psychological truth. There are, indeed, certain acts we agree in calling instinctive, but they are common to man as reason is common to other animals. It is simply a question of degree. Nature does nothing per sallem, and leaves no wide gaps in her ranks. Whatever we may think of the development theory, we can but admit an existing gradation in the mental as well as physical attributes, through the various species of animals and men up to the highest specimens of human kind.

Dr. Lindsay's article is in skeleton, un. clothed here by the numerous facts which he has observed and collected relating to comparative psychology and mental pathology. It is, therefore, impossible to judge of the correctness of his conclusions or the accuracy of his classification. That there is an insanity of animals, there can be as little doubt as that they give evidence of most of the moral and intellectual faculties of man, though in a rudimentary de- 